\title{
Simple and fast evaluation of relaxation parameters of magnetic nanoparticles
}

\author{
Philipp Lemal ${ }^{\mathrm{a}}$, Sandor Balog ${ }^{\mathrm{a}}$, Liliane Ackermann-Hirschi ${ }^{\mathrm{a}}$, Patricia Taladriz-Blanco ${ }^{\mathrm{a}}$, \\ Ann M. Hirt ${ }^{\mathrm{b}}$, Barbara Rothen-Rutishauser ${ }^{\mathrm{a}}$, Marco Lattuada ${ }^{\mathrm{c}, *}$, Alke Petri-Fink ${ }^{\mathrm{a}, \mathrm{c}, *}$ \\ ${ }^{a}$ Adolphe Merkle Institute, University of Fribourg, Chemin des Verdiers 4, CH-1700 Fribourg, Switzerland \\ ${ }^{\mathrm{b}}$ Institut for Geophysics, ETH Zurich, Sonneggstrasse 5, CH-8092 Zurich, Switzerland \\ ${ }^{c}$ Chemistry Department, University of Fribourg, Chemin du Museé 9, CH-1700 Fribourg, Switzerland
}

Keywords:

Magnetic nanoparticles

Magnetic hyperthermia

Nanoparticle characterization

Néel relaxation

Magnetocrystalline anisotropy

\begin{abstract}
A B S T R A C T
The efficacy of magnetic hyperthermia treatment depends on the optimal available magnetic nanoparticles (MNPs) that are excited in a given alternating magnetic field and viscosity of the region of interest. In this regard, assessing the relevant relaxation parameters is of upmost importance and could improve the speed of development of efficient applications. Here, we demonstrate how to deduce all relevant magnetic parameters from fast, independent, and simple experimental measurements such as dynamic light scattering, vibrating sample magnetometer, and lock-in thermography. We study the thermal behaviour of two MNPs with different forms, i.e. spherical and cubical, synthesized in-house by thermal decomposition and coated with 4 different surface agents. By determination of specific absorption rate (SAR) values, hydrodynamic diameters and $M-H$ curves it is possible to compute the magnetic particle volume, dominant relaxation time and magnetocrystalline anisotropy constant. The calculated SAR values derived from these parameters, show good agreement with the experimental determined SAR data, demonstrating the applicability of the reported procedure. Additionally, our results indicate that surface coatings can have minor impacts on the thermal dissipation of Néel relaxation dominated MNPs.
\end{abstract}

\section{Introduction}

Magnetic nanoparticles (MNPs) gained much attention in recent years, due to their biocompatibility [1], biodegradability [2,3], superparamagnetic properties [4], and ability to convert magnetic energy into heat $[1,5,6]$. The conversion of magnetic energy, generated by an alternating magnetic field (AMF), into thermal energy is based on relaxation mechanisms, which can be categorized into Brownian and Néel relaxations [7]. In short, Brownian relaxation describes the complete rotation of the entire particle towards the direction of the applied external magnetic field and generates heat via rotational friction [8], while in Néel relaxation only the magnetic moment inside the particles follows the magnetic field direction and the magnetic energy is dissipated as heat [7]. The efficiency of this conversion is dictated by external parameters including magnetic field frequency [9] and amplitude [10], as well as by several intrinsic particle properties, such as crystallinity [11], surface coating [6], polydispersity index [12], colloidal stability [13], and size [14]. In addition, the latter factor, particle size, determines to a large extent the effective relaxation mechanism
$[7,14]$. Even though the effective relaxation is a combination of both, it is widely accepted that in small MNPs Neel relaxation is the dominant mechanism, while Brownian relaxation dominates for particles with a size above a certain threshold [14]. This value can vary from batch to batch depending upon the magnetocrystalline anisotropy constant, which can be described as the energy needed to change the direction of the dipole moment from the easy axis of magnetization [15]. In short, the higher the magnetocrystalline anisotropy constant for a given particle, the smaller is the nanoparticle size for which the transition from Néel- to Brownian-dominated relaxation occurs [7]. To achieve efficient magnetic hyperthermia treatment, it is of upmost importance to know which is the dominant relaxation mechanism, since Brownian relaxation is hindered at high viscosities and will not dissipate sufficient heat to alter the cells [16]. Hence, the intended area of application and the used magnetic field frequency dictates which relaxation particle type is favored to yield maximum heat dissipation [7]. However, assessing the dominant relaxation mechanism and magneto-crystalline anisotropy constant are challenging and require a combination of several characterization techniques. Brownian relaxation is related to the viscosity

* Corresponding authors at: Adolphe Merkle Institute, University of Fribourg, Chemin des Verdiers 4, CH-1700 Fribourg, Switzerland (A. Petri-Fink).

E-mail addresses: marco.lattuada@unifr.ch (M. Lattuada), alke.fink@unifr.ch (A. Petri-Fink). 
coefficient $\eta$ of the surrounding fluid, the absolute temperature $T$, and the hydrodynamic volume of the nanoparticle $V_{H}$ [14]. While viscosity and temperature are easy to determine, the hydrodynamic particle volume requires more elaborated measurement techniques, e.g. dynamic light scattering (DLS) or Taylor dispersion analysis (TDA) $[17,18]$. In contrast, the characteristic Néel relaxation time is given by the characteristic time $\tau_{0}$ and the ratio of the magnetocrystalline anisotropy constant $K_{V}$ and the magnetic particle volume $V_{p}$ to the absolute temperature $T$ [19]. The value of $\tau_{0}$ is often approximated as $\sim 10^{-9} \mathrm{~s}$ [20], the magnetic particle volume can be estimated from the magnetization $(M-H)$ curve [21], while $K_{V}$ requires more complex experiments and/or calculations based on susceptibility measurements or $M-H$ curves of MNP samples, since its values for nanoparticles often differs substantially from the corresponding bulk material values $[22,23]$.

While it was just recently reported how to evaluate $K_{V}, V_{p}$, and $\tau_{O}$ using the $M-H$ curve and AC susceptibility of immobilized and suspended MNPs [24], we present here a straightforward way to assess the same parameters using simple measurement techniques. In detail, by determining the hydrodynamic diameters via DLS, the $M-H$ curves via vibrating sample magnetometer (VSM), and the specific absorbance rate (SAR) values via lock-in thermography (LIT), we were able to deduce the above-mentioned properties. For that purpose, we synthesized 2 different MNPs batches by thermal decomposition method, transferred them to water, coated each particle batch with 4 different surface agents, and subsequently analysed the most common particle characteristics using simple and fast measurement techniques. The validity of this approach is shown by the good agreement between experimental assessed and modelled SAR values. Furthermore, our results implicate that surface coatings have little impact on the heating efficiency of Néel relaxation dominated MNPs.

\section{Materials and methods}

\subsection{Spions synthesis and coating}

Cubic and spherical superparamagnetic iron oxide nanoparticles (SPIONs) were synthesized by thermal decomposition method described elsewhere $[11,25,26]$. Cubic morphology was achieved by introducing sodium oleate into the reaction $[11,27]$. The obtained particles were transferred into an aqueous environment by a ligand exchange with citric acid (CA) [28]. Catechol-conjugated (Cat-) polymer (Polyethylene glycol, PEG and Polyvinyl alcohol, PVA) was synthesized following a published procedure [29] and dissolved in Milli-Q water. Catechol-PEG was synthesized by a modified procedure from ref. 29. In brief, $6 \mathrm{~mL}$ of dry dimethylformamide (DMF) and $3 \mathrm{~mL}$ of dichloromethane (DCM) were introduced into a balloon. The liquids were degassed with argon and 500 mg PEG-NHS was added to the solution. Subsequently, $95 \mathrm{mg}$ dopamine hydrochloride and trimethylamine were added and the reaction was stirred overnight at RT. CA coated SPIONs were added (10 molecules polymer $/ \mathrm{nm}^{2}$ nanoparticle surface) and the mixtures were sonicated at room temperature overnight. No further purification was done thus keeping the iron concentration constant at $4 \mathrm{mg} / \mathrm{mL}$ for all applied coatings. This procedure was performed for both morphologies and resulted in Cat-PEG $5 \mathrm{kDa}$, Cat-PEG $2 \mathrm{kDa}$, and Cat-PVA $5 \mathrm{kDa}$ coated SPIONs.

\subsection{Particle analysis}

The iron content of CA coated SPIONs was analysed according to a procedure published elsewhere [12] using a PerkinElmer Avio 200 inductively coupled plasma optical emission spectroscopy (ICP-OES). Diluted samples of CA-coated cubic and spherical SPIONs were prepared for transmission electron microscopy (TEM) by a reported procedure [30]. In brief, by mixing MNPs with a defined concentration of bovine serum albumin (BSA), the individual particles get stabilized against aggregation and drying artefacts are avoided. TEM images were collected with a side-mounted Olympus Veleta CCD camera using a FEI Tecnai Spirit, operating at a voltage of $120 \mathrm{kV}$. X-ray diffractograms (XRD) were collected with an Ultima IV X-Ray diffractometer from Rigaku. The dried samples were measured between $20^{\circ}$ and $80^{\circ}$ and the resulting diffractograms were normalized to show the same maximum peak height. Zeta potential measurements were estimated via the Henry equation using the Smoluchowski approximation and measured at $25^{\circ} \mathrm{C}$ in a Milli-Q water/PBS mixture using a Brookhaven ZetaPALS. Dynamic light scattering (DLS) experiments were carried out on a 3D LS spectrometer of LS Instruments at $25{ }^{\circ} \mathrm{C}$ as described elsewhere [11]. To assess the magnetic properties, coated nanoparticle suspensions were deposited onto hydrophobic cotton pellets and dried. Vibrating sample magnetometer (VSM) measurements were carried out on a Model 3900 VSM of Princeton Measurements Corporation. Magnetization curves were measured at room temperature and normalized by the dried sample mass of iron. All thermal measurements of nanoparticle dispersions were conducted with an in-house developed lock-in thermal (LIT) imaging set-up [31,32]. MNP heating was induced in an alternating magnetic field of $523 \mathrm{kHz}-10.45 \mathrm{mT}, 331 \mathrm{kHz}-7.62 \mathrm{mT}$, $173 \mathrm{kHz}-6.04 \mathrm{mT}$, and $110 \mathrm{kHz}-5.97 \mathrm{mT}$, respectively, and recorded with an infrared camera. VSM and LIT data were analysed by the Shapiro-Wilk normality test and their significance level determined using the two-sample $t$-test.

\subsection{Modelling of heat dissipation}

Linear response theory (LRT) was used to interpret the heat generated by SPIONs exposed to an alternating magnetic field. According to this theory, the power $P$ generated per unit mass of particles exposed to a magnetic field with an amplitude $H_{0}$, frequency $f$ :

$$
\begin{gathered}
P=\pi \mu_{0} H_{0}^{2} f \chi_{0} \frac{2 \pi f \tau}{1+(2 \pi f \tau)^{2}} \\
\chi_{0}=\frac{\mu_{0} M_{S}^{2} V_{p}}{3 \rho k T} \frac{3}{\xi}\left(\operatorname{coth}(\xi)-\frac{1}{\xi}\right) \\
\xi=\frac{\mu_{0} M_{s} V_{p} H_{0}}{k T} \\
\frac{1}{\tau}=\frac{1}{\tau_{B}}+\frac{1}{\tau_{N}} \Rightarrow\left\{\begin{array}{c}
\tau_{B}=\frac{3 \eta V_{H}}{k T} \\
\tau_{N}=\frac{\sqrt{\pi}}{2} \tau_{0} \frac{\exp (\sigma)}{\sqrt{\sigma}} \\
\sigma=\frac{K_{V} V_{p}}{k T}
\end{array}\right.
\end{gathered}
$$

In the above equation, $M_{s}$ is the saturation magnetization of particles, which is obtained from magnetization data, $V_{p}$ is the particle magnetic volume, $k$ is Boltzmann constant, $\mu_{0}$ is the vacuum magnetic permeability, $T$ the absolute temperature, $\tau$ is the overall relaxation time (combination of Brownian and Néel relaxation times), $\tau_{0}$ is the characteristic internal relaxation time of the material, which is often assumed to be $\sim 10^{-9} \mathrm{~s}, V_{H}$ is the hydrodynamic volume of particles, $\eta$ is the viscosity of the solution, $K_{V}$ is the magnetocrystalline anisotropy constant and $\rho$ is the particle density. While the hydrodynamic volume of the particles is determined from DLS data, the magnetic volume can only be determined by looking at magnetization curves. As already suggested in the literature, we have assumed that, in spite of the narrow size distribution of particles as seen from TEM images, the prepared SPIONs can still have a broader distribution of magnetic size, and consequently of magnetic volumes, due to defects in the crystalline structure and to the existence of magnetic dead layer. Therefore, we have assumed that it is possible to describe the magnetic size distribution by means of the lognormal function. We have further assumed that each particle volume has a magnetization behaviour that can be described by means of a Langevin function. Therefore, the magnetization curve of the particles can be described as follow: 

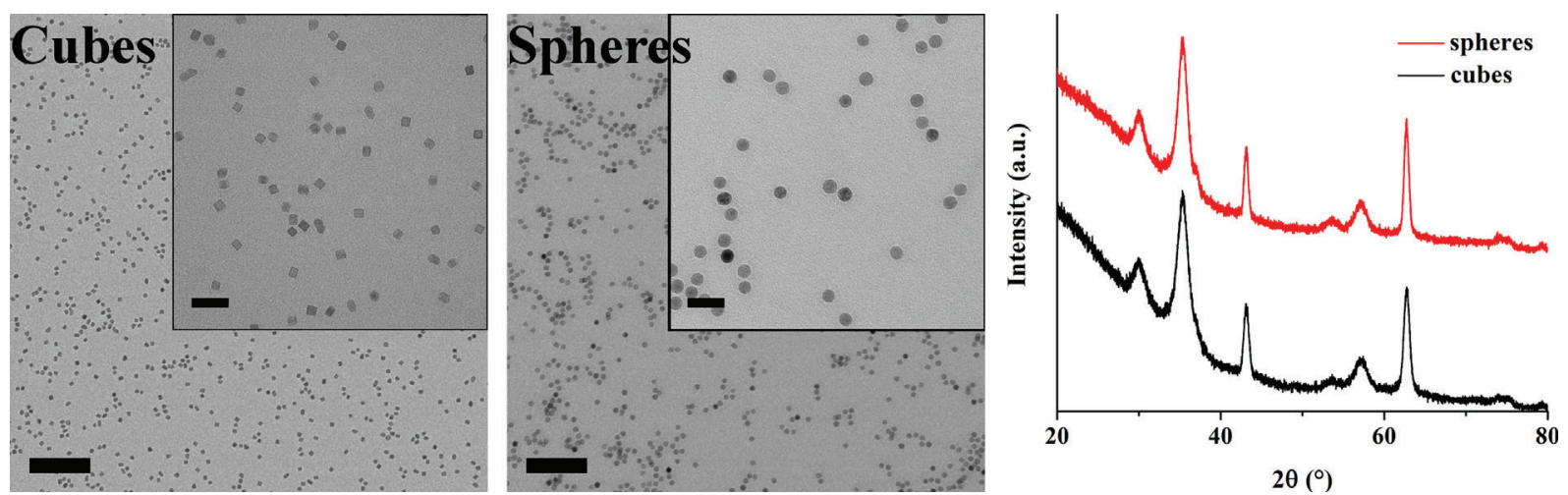

Fig. 1. TEM micrographs of CA coated SPIONs and corresponding x-ray diffractograms. Scale bar $=200 \mathrm{~nm}$, insets $=50 \mathrm{~nm}$.

$$
\begin{gathered}
M(B)=M_{s} \frac{\int g(r) r^{3}\left(\operatorname{coth}(x)-\frac{1}{x}\right) d r}{\int g(r) r^{3} d r} \\
x=\frac{4 \pi r^{3} M_{s} B}{3 k_{b} T} \\
g(r)=\frac{1}{\sqrt{2 \pi} r \cdot s} \exp \left(-\frac{(\log (r)-m)^{2}}{2 s^{2}}\right)
\end{gathered}
$$

In the above equation, $\mathrm{B}$ is the intensity of the applied magnetic field, $g(r)$ is the lognormal magnetic particle size distribution, with $s$ and $m$ being the mean and standard deviation of the natural logarithm of the size $r$. A fitting of the magnetization curves, allowing us to retrieve the two parameters $s$ and $m$ for the each SPIONs sample, is shown in the supporting information (Fig. S3).

\section{Results and discussion}

Cubic and spherical SPIONs were synthesized in house following the well-known thermal decomposition method [11,25,26] and subsequently transferred to water by ligand exchange with CA [28]. Fig. 1 shows that the particles are well dispersed after the ligand exchange and are entirely composed of magnetite/maghemite. The diameter was found to be $14.3 \pm 2.0 \mathrm{~nm}$ for the cubes and $18.7 \pm 2.4 \mathrm{~nm}$ for the spheres (determined using ImageJ particle sizing software, $n>1000$ nanoparticles).

Furthermore, zeta potential and DLS measurements confirm the colloidal stability of the CA coated particles (Table 1) and hence, were the starting point for all further functionalization. For that purpose, catechol-conjugated polymers were dissolved in Milli-Q water and cubic and spherical SPIONs, respectively, were added to the polymer solutions and sonicated at room temperature overnight. To ensure comparable results, we choose the same particle concentration in all dispersions, performed all coatings at the same time using the exact same parameters and also included the CA coated SPIONs into the sonication process. All data presented in this work, including the CA coated SPIONs, represent the results obtained after overnight sonication. The success of each individual coating process is displayed in Table 1. As can be expected, the respective hydrodynamic diameters increase upon coating the particles with the $5 \mathrm{kDa}$ polymers, whereas the shorter $2 \mathrm{kDa}$ PEG resulted in hydrodynamic sizes comparable to CA SPIONs. However, the increased zeta potential proves successful coating since the strongly negative citric acid is partially replaced by the grafted (neutral) polymer. Additionally, DLS shows that no aggregation occurred upon polymer coating (Fig. S1, supplementary information), which allowed us to study the effect of different coatings of single MNPs on heating.

In a second step, we analysed the MNPs' magnetic and thermal properties (Fig. 2). All particles display superparamagnetic behaviour and no hysteresis could be detected in the $M-H$ curve (Fig. S2). Fig. 2 shows that the normalized (by iron mass) magnetization of spherical particles is lower than the magnetization of cubic MNPs. This can be explained with shape effects, resulting in an increased magnetic core volume and a lowered surface to volume ratio for quasi-cubic particles [33], as well as the synthesis process, which leads to paramagnetic impurities in larger particles [34]. Nonetheless, regarding the effect of coating, no significant difference in magnetization can be observed, neither between the 4 different coated cubic particles nor between the spherical particles coatings which is in good agreement with previous reported results [35]. In contrast, the SAR values for all three polymer coated spheres were found to be statistically significant lower than the SAR value of the CA spheres. This behaviour was only partially observable for the cubes, where the Catechol-PEG $5 \mathrm{kDa}$ show a significant decrease in SAR to CA coated cubes, while Catechol-PEG $2 \mathrm{kDa}$ and Catechol-PVA $5 \mathrm{kDa}$ are not significantly different. These small changes might be attributed to the overall lower SAR values of the cubes due to their smaller size [12].

In order to obtain a better understanding of the heating behaviour of cubic and spherical MNPs, we decided to investigate the heat dissipation mechanism in more detail. We used linear response theory [14] in order to compute the heat generated by the MNPs. It is well known that the magnetic particle size distribution plays a central role in the heat generated. Therefore, we first used magnetization data to estimate the magnetic particle size distribution, which is different from the crystallite size distribution determined via TEM. The calculation has been

Table 1

\begin{tabular}{|c|c|c|c|c|c|}
\hline Particle type & Coating & Zeta potential $(\mathrm{mV})$ & $\mathrm{d}_{H, D L S}(\mathrm{~nm})$ & $\tau_{0}(\mathrm{~s})$ & $\mathrm{K}_{V}\left(\mathrm{~J} / \mathrm{m}^{3}\right)$ \\
\hline \multirow[t]{4}{*}{ Cubes } & Citric Acid & $-26.4 \pm 0.6$ & $19.2 \pm 0.2$ & $1 \cdot 10^{-9}$ & 4587 \\
\hline & Catechol-PEG $2 \mathrm{kDa}$ & $-4.5 \pm 1.1$ & $19.0 \pm 0.2$ & $1 \cdot 10^{-9}$ & 3595 \\
\hline & Catechol-PEG $5 \mathrm{kDa}$ & $-6.6 \pm 0.6$ & $32.4 \pm 0.8$ & $1 \cdot 10^{-9}$ & 3919 \\
\hline & Catechol-PVA $5 \mathrm{kDa}$ & $-4.7 \pm 0.6$ & $27.8 \pm 0.4$ & $1 \cdot 10^{-9}$ & 3527 \\
\hline \multirow[t]{4}{*}{ Spheres } & Citric Acid & $-27.2 \pm 0.8$ & $23.0 \pm 0.2$ & $6 \cdot 10^{-9}$ & 6205 \\
\hline & Catechol-PEG $2 \mathrm{kDa}$ & $-4.4 \pm 0.9$ & $23.2 \pm 0.2$ & $6 \cdot 10^{-9}$ & 5007 \\
\hline & Catechol-PEG $5 \mathrm{kDa}$ & $-5.2 \pm 0.5$ & $34.0 \pm 2.4$ & $6 \cdot 10^{-9}$ & 6137 \\
\hline & Catechol-PVA $5 \mathrm{kDa}$ & $-4.3 \pm 1.4$ & $31.2 \pm 0.2$ & $6 \cdot 10^{-9}$ & 5749 \\
\hline
\end{tabular}

Zeta potential and hydrodynamic diameter determined by DLS as a function of particle type and applied coatings. Characteristic time and magnetocrystalline anisotropy constant calculated from experimental data as a function of particle type and applied coating. 

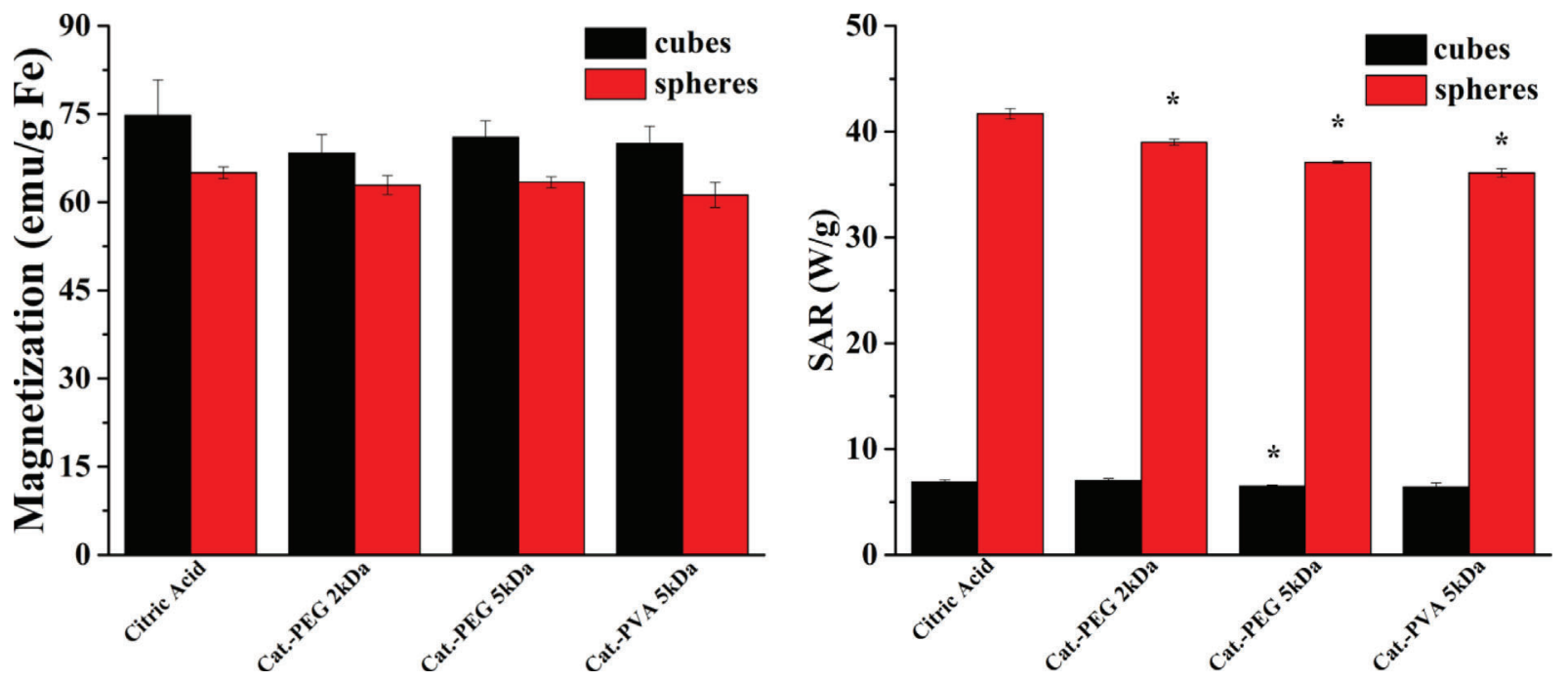

Fig. 2. Saturation magnetization (left) and SAR values (right) as a function of particle morphology and applied coating. Saturation magnetization values show no significant differences upon coating for both particle types. Spheres exhibit a significant difference* in SAR values between polymer coated and CA coated SPIONs whereas cubes show only a significant difference* for Catechol-PEG $5 \mathrm{kDa}$ coating. Values were considered significant compared with their respective CA coated SPIONs when $\mathrm{p}<0.05$.

made under the assumption that the magnetization curve of particles with a given magnetic size follows the Langevin equation. By assuming that the magnetic size distribution can be described effectively by a lognormal distribution, the two parameters of the distribution have been obtained by fitting the magnetization data. With the knowledge of the magnetic size distribution, we have performed calculation using LRT, and used the magnetocrystalline anisotropy constant and the relaxation time constant $\tau_{0}$ as adjustable parameters to fit the experimental SAR data. In order to minimize the uncertainty in these parameters and precisely determine which the dominant relaxation mechanism is, we have performed for all the spherical samples SAR measurements at four different field frequency values. We have found that the small dependence of the SAR value to the change in surface coating shown in Fig. 2 can only be explained by assuming that Brownian relaxation is not the dominant mechanism of heat dissipation in our system. However, Néel relaxation is the prevailing mechanism, which is supported by the low value of the magnetocrystalline anisotropy constant (Table 1) that can be used to model the experimental SAR data. The corresponding values of the relaxation time $\tau_{0}$ are for all samples of spherical particles about $6 \cdot 10^{-9} \mathrm{~s}$. With these values, the heating behaviour of all spherical SPIONs can be quantitively accounted for, as shown in Fig. 3.

In the case of cubes, a similar analysis can be carried out. Because their size regime is in the area of Néel dominated relaxation time, we determined SAR values at only one frequency to show the simplicity of this approach. It is found that using relaxation time value of $10^{-9} \mathrm{~s}$, values of the magnetocrystalline anisotropy constant are ca. $3500-4500 \mathrm{~J} / \mathrm{m}^{3}$, depending on the type of coating, lead to results in good agreement with the experimental data.

In conclusions, the results of our calculations indicate that the low magnetocrystalline anisotropy constant values found for all nanoparticles cause Néel relaxation to be the dominant heat dissipation mechanism, which explains the small differences in SAR values observed for all particles. In addition, the small differences in SAR values appear to be related to the little differences in the magnetocrystalline anisotropy values for the particles bearing different coatings.

One important aspect should be noted, however. There is a significant difference between the SAR values of particles measured a day after preparation at a frequency of $523 \mathrm{kHz}$, reported in Fig. 2, and the same particles measured a few weeks after preparation at various frequencies (including $523 \mathrm{kHz}$ ), reported in Fig. 3. This suggests that the
SAR values decrease in time after preparation, and the calculations indicate that the magnetocrystalline anisotropy constant is lower directly after preparation $\left(K_{v}=1700-2100 \mathrm{~J} / \mathrm{m}^{3}\right)$ than after a few weeks $\left(K_{v}=5000-6200 \mathrm{~J} / \mathrm{m}^{3}\right)$. Such change might be attributed to aging of MNPs in the presence of oxygen, resulting in oxidation of magnetite to maghemite even inside the nanoparticle core $[36,37]$. The conclusion of dominant Néel relaxation mechanism applies anyway to all datasets.

\section{Conclusions}

We have presented the derivation of the magnetocrystalline anisotropy constant and effective relaxation mechanism of MNPs from simple, independent, and fast measurement techniques. We demonstrated the applicability of this approach using two SPIONs batches, synthesized in-house by thermal decomposition method, and applied four different surface coatings. By acquiring their $M-H$ curves, SAR values, and hydrodynamic diameters it is possible to calculate all relevant parameters needed to assess the magnetic properties which are imperative for magnetic hyperthermia. Hence, a simple and reliable quantification of the dominant relaxation time and magnetocrystalline anisotropy constant can be useful for a better understanding of the areas of application and could facilitate the development of more efficient magnetic hyperthermia treatments. Additionally, our results demonstrate the influence of surface coatings on the heating efficiency of Néel relaxation dominated MNPs. Minor changes in SAR values attributed to the different coatings can be observed, based on changed magnetocrystalline anisotropy constants.

\section{Declaration of Competing Interest}

There is no conflict of interest.

\section{Acknowledgements}

The authors gratefully acknowledge the financial support from the Swiss National Science Foundation (159803), the University of Fribourg and the Adolphe Merkle Foundation. Parts of this work were supported by the National Center of Competence in Research (NCCR) for BioInspired Materials. Access to VSM was amicably earmarked by the Institute for Geophysics of the ETH Zurich. Utilization of XRD was kindly provided by the Unit of Earth Sciences, Department of 

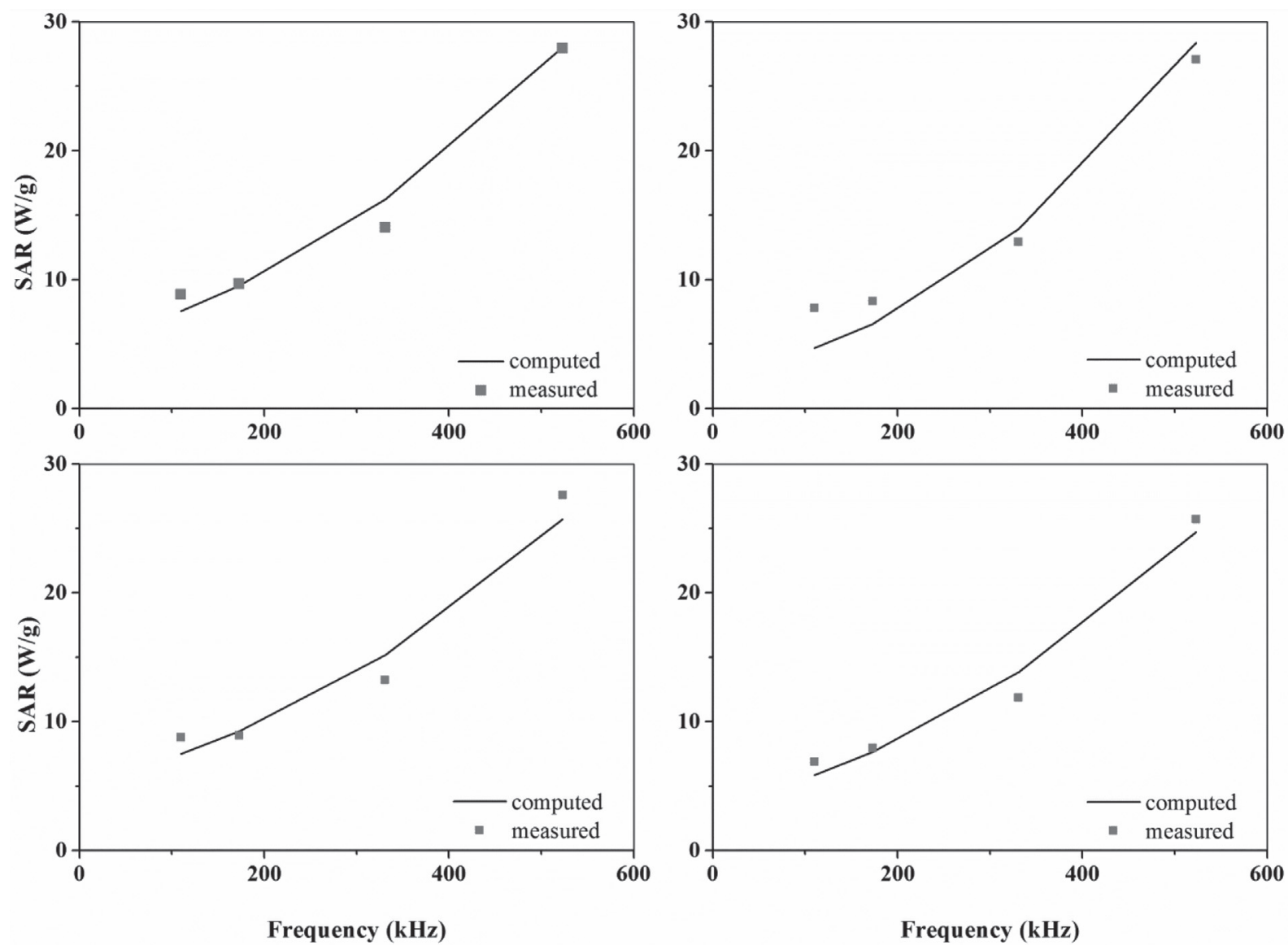

Fig. 3. Comparison between experimental and computed SAR data of spheres as a function of applied coating and the magnetic field frequency.

Geosciences of the University of Fribourg.

\section{Appendix A. Supplementary data}

Supplementary data to this article can be found online

\section{References}

[1] E.A. Périgo, et al., Fundamentals and advances in magnetic hyperthermia, Appl. Phys. Rev. 2 (2015) 041302-1-35.

[2] H. Markides, et al., Biocompatibility and toxicity of magnetic nanoparticles in regenerative medicine, J. Nanomater. (2012) 1-11.

[3] K. Desboeufs, et al., Massive intracellular biodegradation of iron oxide nanoparticles evidenced magnetically at single-endosome and tissue levels, ACS Nano 10 (2016) 7627-7638.

[4] Z. Hedayatnasab, et al., Review on magnetic nanoparticles for magnetic nano fluid hyperthermia application, Mater. Des. 123 (2017) 174-196.

[5] S. Laurent, et al., Magnetic fluid hyperthermia: focus on superparamagnetic iron oxide nanoparticles, Adv. Colloid Interface Sci. 166 (1-2) (2011) 8-23.

[6] A.K. Gupta, M. Gupta, Synthesis and surface engineering of iron oxide nanoparticles for biomedical applications, Biomaterials 26 (18) (2005) 3995-4021.

[7] A.E. Deatsch, B.A. Evans, Heating efficiency in magnetic nanoparticle hyperthermia, J. Magn. Magn. Mater. 354 (2014) 163-172.

[8] S. Noh, et al., Recent advances of magneto-thermal capabilities of nanoparticles from design principles to biomedical applications, Nano Today 13 (2017) 61-76.

[9] W.J. Atkinson, et al., Usable frequencies in hyperthermia with thermal seeds, IEEE Trans. Biomed. Eng. BME-31 (1) (1984,) 70-75.

[10] B. Kozissnik, et al., Magnetic fluid hyperthermia: advances, challenges, and opportunity, Int. J. Hyperthermia 29 (8) (2013) 706-714.

[11] P. Lemal, et al., Heating behavior of magnetic iron oxide nanoparticles at clinically relevant concentration, J. Magn. Magn. Mater. 474 (2019) 637-642.

[12] P. Lemal, et al., Lock-in thermography as a rapid and reproducible thermal characterization method for magnetic nanoparticles, J. Magn. Magn. Mater. 427 (2016) 206-211.

[13] C. Kumar, F. Mohammad, Magnetic nanomaterials for hyperthermia-based therapy and controlled drug delivery, Adv. Drug Deliv. Rev. 63 (9) (2011) 789-808.

[14] R. Rosensweig, Heating magnetic fluid with alternating magnetic field, J. Magn.
Magn. Mater. 252 (2002) 370-374.

[15] S. Oyarzún, et al., Size effects in the magnetic anisotropy of embedded cobalt nanoparticles: from shape to surface, Sci. Rep. 5 (2015) 16-21.

[16] N. Hervault, et al., Magnetic nanoparticle-based therapeutic agents for thermochemotherapy treatment of cancer, Nanoscale 6 (2014) 11553-11573.

[17] S. Balog, et al., Characterizing nanoparticles in complex biological media and physiological fluids with depolarized dynamic light scattering, Nanoscale 7 (14) (2015) 5991-5997.

[18] P. Lemal, et al., Nanoparticles and taylor dispersion as a linear time-invariant system, Anal. Chem. 91 (2018) 1217-1221.

[19] R. Hergt, et al., Magnetic particle hyperthermia: nanoparticle magnetism and materials development for cancer therapy, J. physics. Condens. matter 18 (2006) S2919-S2934.

[20] A. Muxworthy, W. Williams, Critical superparamagnetic/single-domain grain sizes in interacting magnetite particles: implications for magnetosome crystals, J. R. Soc. Interface 6 (2009) 1207-1212.

[21] P. Bender, et al., Distribution functions of magnetic nanoparticles determined by a numerical inversion method, New J. Phys. 19 (2017) 073012-1-19.

[22] G.T. Landi, et al., AC susceptibility as a tool to probe the dipolar interaction in magnetic nanoparticles, J. Magn. Magn. Mater. 421 (2017) 138-151.

[23] A. Elrefai, T. Sasayama, Empirical expression for DC magnetization curve of immobilized magnetic nanoparticles for use in biomedical applications, AIP Adv. 8 (2018) 056803-1-6.

[24] A.L. Elrefai, et al., Magnetic parameters evaluation of magnetic nanoparticles for use in biomedical applications, J. Magn. Magn. Mater. 474 (2019) 522-527.

[25] J. Park, et al., Ultra-large-scale syntheses of monodisperse nanocrystals, Nat. Mater 3 (12) (2004) 891-895.

[26] Y. Hou, et al., Controlled synthesis and chemical conversions of $\mathrm{FeO}$ nanoparticles, Angew. Chemie - Int. Ed. 46 (33) (2007) 6329-6332.

[27] E. Wetterskog, et al., Precise control over shape and size of iron oxide nanocrystals suitable for assembly into ordered particle arrays, Sci. Technol. Adv. Mater. 15 (2014) 055010.

[28] M. Lattuada, T.A. Hatton, Functionalization of monodisperse magnetic nanoparticles functionalization of monodisperse magnetic nanoparticles, Langmuir 23 (4) (2007) 2158-2168.

[29] D. Burnand, et al., Catechol-derivatized poly(vinyl alcohol) as a coating molecule for magnetic nanoclusters, J. Magn. Magn. Mater. 380 (2015) 157-162.

[30] B. Michen, et al., Avoiding drying-artifacts in transmission electron microscopy: characterizing the size and colloidal state of nanoparticles, Sci. Rep. 5 (2015) 9793.

[31] C.A. Monnier, et al., Lock-in thermography as an analytical tool for magnetic 
nanoparticles: measuring heating power and magnetic fields, J. Phys. Chem. C 121 (2017) 27164-27175.

[32] C.A. Monnier, et al., A lock-in-based method to examine the thermal signatures of magnetic nanoparticles in the liquid, solid and aggregated states, Nanoscale 8 (2016).

[33] G. Kandasamy, D. Maity, Recent advances in superparamagnetic iron oxide nanoparticles (SPIONs) for in vitro and in vivo cancer nanotheranostics, Int. J. Pharm. 496 (2) (2015) 191-218.
[34] A. Lak, et al., Size dependent structural and magnetic properties of $\mathrm{FeO}-\mathrm{Fe} 3 \mathrm{O} 4$ nanoparticles, Nanoscale 5 (2013) 12286-12295.

[35] L. Gutiérrez, et al., Aggregation effects on the magnetic properties of iron oxide colloids, Nanotechnology 30 (11) (pp, 2019.) 14.

[36] C. Rümenapp, et al., Monitoring of the aging of magnetic nanoparticles using Mössbauer spectroscopy, J. Magn. Magn. Mater. 380 (2015) 241-245.

[37] L.K. Bogart, et al., Environmental oxidative aging of iron oxide nanoparticles, Appl. Phys. Lett. 113 (2018) 133701-1-4. 